\title{
Rapid analysis of fungal membrane lipids and their isotopic composition by TMSH/TMAH derivatization Pyrolysis coupled GC-MS/IRMS analysis
}

\author{
STANISLAV JABINSKI ${ }^{1,2}$, WESLEY DE MELO RANGEL ${ }^{2}$, \\ FRANTISEK LORENC ${ }^{2}$ AND TRAVIS MEADOR ${ }^{1,2}$ \\ ${ }^{1}$ University of South Bohemia \\ ${ }^{2}$ Biology Center CAS \\ Presenting Author: stanislav.jabinski@bc.cas.cz
}

Each year, roughly one-sixth of atmospheric carbon (115Pg C) is cycled through terrestrial vegetation, channelled into the soils below, and respired back to $\mathrm{CO}_{2}$. However, predicting the reactivity and $\mathrm{CO}_{2}$ buffering capacity of soil systems and thus the carbon-climate feedback in terrestrial ecosystems is limited by poor understanding of the accessibility and turnover of "recalcitrant" organic matter in soils. As major decomposers in soil ecosystems, fungi are among the few organisms on the planet that can feed on chemically stable forms of organic $\mathrm{C}$ but are also known to directly access recently produced plant photosynthate, but their role in soil ecosystems is still poorly understood. To further delineate fungal activity and substrate turnover, this study aims to determine fungal metabolic modes and rates based on the absolute and relative assimilation rates of $\mathrm{D}_{2} \mathrm{O}$ and ${ }^{13} \mathrm{C}$-bicarbonate into fungal biomass, fatty acids, and sterols.

Recently, dual stable isotope probing (Dual-SIP) methods have been developed to track microbial assimilation of heavy water $\left(\mathrm{D}_{2} \mathrm{O}\right)$ and ${ }^{13} \mathrm{C}$-bicarbonate into membrane lipids in order to provide estimates of total activity and anabolic $\mathrm{C}$-uptake fluxes and can be advantageously performed without altering soil conditions. We employ this approach to establish the fundamental relationships between fungal ecotypes and their food sources (explanatory variables) on water isotopic fractionation [ $\delta \operatorname{lipid} / \mathrm{H}_{2} \mathrm{O}$, water assimilation factor $\left.\left(\mathrm{a}_{\mathrm{w}}\right)\right]$, and anaplerotic incorporation of inorganic $\mathrm{C}$ into fungal fatty acids and ergosterol (response variables). A culturing pipeline was established to incubate different fungal ecotypes with different substrates ranging from monosaccharides to complex molecules to identify ecotype- and substrate-specific isotopic signatures. Pyrolysis gas chromatography mass spectrometry (Pyr-GC-MS) products give fingerprint information of SOM and biomass at the molecular level, allowing for the characterization and quantification of compound-specific molecules without the requirement of lab intensive wet chemistry protocols. Here we implement a protocol for rapid analysis of fungal membrane biomarkers and their isotopic composition by a well known Tetramethylammonium hydroxide (THAM) and Trimethylsulfonium hydroxide (THSM) derivatization approach prior to the Pyr-GC-MS/IRMS analysis for hydrogen isotopes. 